\title{
Accuracy and precision of fixation locations recorded with the low-cost Eye Tribe tracker in different experimental set- ups
}

\author{
Kristien Ooms \\ Ghent University \\ Lieselot Lapon \\ Ghent University
}

\author{
Lien Dupont \\ Ghent University \\ Stanislav Popelka \\ Palacký University Olomouc
}

\begin{abstract}
This article compares the accuracy and precision of the low-cost Eye Tribe tracker and a well-established comparable eye tracker: SMI RED 250. Participants were instructed to fixate on predefined point locations on a screen. The accuracy is measured by the distance between the recorded fixation locations and the actual location. Precision is represented by the standard deviation of these measurements. Furthermore, the temporal precision of both eye tracking devices (sampling rate) is evaluated as well. The obtained results illustrate that a correct set-up and selection of software to record and process the data are of utmost importance to obtain acceptable results with the low-cost device. Nevertheless, with careful selections in each of these steps, the quality (accuracy and precision) of the recorded data can be considered comparable.
\end{abstract}

Keywords: low-cost eye tracker, accuracy, precision, eye tracker set-up evaluation, fixation detection algorithm

\section{Introduction}

The use of eye tracking - especially in scientific research - is not new. It has been around since the end of the $19^{\text {th }}$ century (e.g. Buswell, 1935; Dodge \& Cline, 1901). However in the past, this was often an expensive method due to equipment costs and time consuming process of analyzing the huge amounts of recorded data (Jacob \& Karn, 2003). Some authors in that period concluded that we cannot learn much from eye tracking data (e.g. Tinker, 1946). Near the end of the 1960s, the importance of eye movement recordings - and the visualization hereof - was illustrated by Yarbus (Borji \& Itti, 2014; Yarbus, 1967). Due to, on the one hand, the improvements of the eye tracking systems themselves (becoming easier to operate, less intrusive and more reliable), and advances in related psychological theories, on the other hand, a rise in eye tracking studies was noticed in scientific research in the 1970s (e.g. Just \& Carpenter, 1976; Rayner, 1998).

By the 1980s, eye tracking was integrated in research regarding Human Computer Interaction (HCI). This does not only involve user studies to detect and evaluate issues in the interactions between humans and computers (e.g. usability engineering); eye tracking is also used in real time as input device. In the 1990s, the computer systems themselves have become more interactive with the rise of the Internet, use of website, email, videoconferencing, etc. (e.g. Jacob \& Karn, 2003; Rayner \& Castelhano, 2008; Schiessl, Duda, Thölke, \& Fischer, 2003; Zambarbieri, Carniglia, \& Robino, 2008).

Nowadays, eye tracking experiments are becoming more common in different research fields, not only in psychology: e.g. usability engineering, cartography, landscape research, marketing, sport and movement sciences (Akinlofa, Holt, \& Elyan, 2014; Dupont, Antrop, \& Van Eetvelde, 2014; Ooms, De Maeyer, Fack, Van Assche, \& Witlox, 2012; Pieters, 2008; Vansteenkiste, Cardon, 
D'Hondt, Philippaerts, \& Lenoir, 2013). Over the years, the accuracy of the equipment has improved and prices have dropped. Furthermore, more advanced analytical processing tools are available, both in terms of software (e.g. visual analytics, data mining) and hardware (e.g. capacity to store data, CPU speed, network performance). The fast technological advancements have been used to produce a new breed of eye trackers; assembled from readily available parts such as webcams and IR-LED emitters.

Because of the now already long standing history of eye tracking, researchers have gained sufficient insights in how these systems work. The previous elements combined resulted in the appearance of 'homemade eye tracking systems', such as the ones presented by Berces and Török (2013); Mantiuk, Kowalik, Nowosielski, and Bazyluk (2012). The newest emerging trend is the promotion of conducting online eye tracking experiments through the participants' own webcam (e.g. EyeSee, 2015; GazeHawk, 2015; XLab, 2015). Together with open source platforms for recording and processing the data (e.g. Dalmaijer, Mathôt, \& Van der Stigchel, 2014; Krassanakis, Filippakopoulou, \& Nakos, 2014; Voßkühler, Nordmeier, Kuchinke, \& Jacobs, 2008), eye tracking can 'in theory' nowadays be conducted without any costs. Furthermore, these cheap (and small) devices have a number of other advantages in comparison to the solutions of the traditional eye tracking vendors (such as SMI, SR Research, Tobii, etc.):

- Transportability: small sized eye trackers are much easier to transport. Furthermore, because of their limited cost one is more inclined to take the risk of moving the device. This means that more participants can be tested outside the lab, in their natural environment;

- Using multiple devices: because of their limited costs, researchers can afford to buy multiple eye trackers. This means that more experiments can be executed, possibly in parallel.

These advantages result in the fact that more participants can be reached in a more realistic setting, which is often an issue in eye tracking research. This would mean a giant leap forward in scientific research related to eye tracking. Nevertheless, these systems are useless to science if their accuracy and precision decreases with the same factor as their price, compared to the wellestablished systems such as from SMI, Tobii,
SR Research which have already proven their value in many scientific research fields. For example, the cost of a SMI RED 250 or Tobii T60 \& T120 eye tracker (two comparable systems) is currently around 30000 euros (depending on which recording and analysis packages are included). The company 'The Eye Tribe' promotes their eye tracker to be "The world's first $\$ 99$ eye tracker with full SDK" (TheEyeTribe, 2015a). When ordering this device, some taxes and transport costs are added, but the final cost is still only around 250 euros.

The aim of this paper is to evaluate one 'cheap' eye tracking system - The Eye Tribe eye tracker - by comparing its accuracy, precision with that of a comparable well-established (and more expensive) eye tracking system (SMI RED 250) in different experimental set-ups. This includes, among others, variations in positioning the devices, sampling rate, fixation detection algorithms, recording software, etc. The justification of the selection of the specific systems is described in the section 'Methods' and 'Study Design'.

\section{Factors in Data Quality}

When evaluating an eye tracking system, one is typically interested in the quality of the (raw) data that it produces. Data quality is a complex issue which is influenced by many factors, such as the properties and characteristics of the eye tracking device itself, but also those of the participants, calibration procedure, environment in which the study was conducted (e.g. lightening conditions), application of filters, task, experiment set-up (relative position of devices), etc. (Blignaut, Holmqvist, Nyström, \& Dewhurst, 2014; Holmqvist et al., 2011; Holmqvist, Nyström, \& Mulvey, 2012; Nyström, Andersson, Holmqvist, \& van de Weijer, 2013). Furthermore, there are currently no standards regarding what should be reported, as some properties are dependent of, for example, the task that should be executed.

One of the best documented characteristics of an eye tracking device is its sampling frequency or sampling rate. A distinction is typically made between high-speed and low-speed systems (Holmqvist et al., 2011). Although there is no clear line between these two types of systems, a sampling rate of $250 \mathrm{~Hz}$ is typically reported as being the minimum for high-speed systems. Highspeed systems are more expensive and result in more measurements per second. This is important when applying event detection algorithms. High-speed systems are 
desirable to be able to detect fast eye movements, but are not necessary when analyzing eye movements at a low speed.

The most important aspects of data quality are accuracy and precision (Blignaut et al., 2014; Holmqvist et al., 2011; Holmqvist et al., 2012). Accuracy is defined as the difference between the true gaze position (screen coordinates of the fixation points) and the recorded fixation positions. The distance between both positions is called offset. Most often, an artificial eye is used to produce reliable and controlled eye movements. Precision refers to the device's ability to reliably produce gaze positions, both regarding the spatial and temporal aspect. Spatial precision can thus be expressed by reporting the standard deviation of the measured offsets (Blignaut et al., 2014; Holmqvist et al., 2011; Holmqvist et al., 2012; Nyström et al., 2013; Reingold, 2014; Wass, Forssman, \& Leppänen, 2014).

Besides the sampling rate, manufactures also report the theoretical accuracy of their devices. However, several authors found that the reported values (most often < $0.5^{\circ}$ ) are too optimistic (and are actually closer to $1.0^{\circ}$ ) (Blignaut et al., 2014; Nyström et al., 2013).

To evaluate the quality of eye movement recordings, raw data is most often considered because fixations are a result of an additional processing on the origin data. These event detection algorithms can take different forms, but only dispersion-based algorithms can be applied on low-speed recordings. Even within the category of dispersion based algorithms, variations exist. Consequently, the resulting data quality is dependent on these algorithms and their associated parameters (Holmqvist et al., 2011; Holmqvist et al., 2012; Salvucci \& Goldberg, 2000).

Fixation detection is an essential element of the whole experimental set-up and should thus not be ignored when evaluating data quality. Nyström et al. (2013), for example, illustrate the influence of precision on the fixation (number and duration).
Ooms, K., Dupont, L., Lapon, L., \& Popelka, S. (2015) Evaluation of the accuracy and precision of a low-cost eye tracking device

\section{Methods}

\section{Extension of Current Research}

We are not the first to express our concerns regarding this new trend. In his paper, Dalmaijer (2014) evaluated the question "Is the low-cost EyeTribe eye tracker any good for research?". He concluded that the Eye Tribe tracker can be appropriate for scientific research related to fixation analyses and pupillometry, but not for evaluating high accuracy saccadic metrics. This latter issue is related to the low sampling rate of $60 \mathrm{~Hz}$.

Although these are promising findings, we do have a number of critics on the evaluation procedure that was applied in this study. First, the Eye Tribe tracker was compared with an EyeLink1000 eye tracker of SR Research. This is undoubtedly a very accurate system as participants need to place their head on a chin rest and their eyes are tracked with a sampling rate of $1000 \mathrm{~Hz}$ (or once every $\mathrm{ms}$ ). This means that the overall settings of this eye tracking system are not comparable with those of the Eye Tribe tracker. The issues are:

- The Eye Tribe tracker is typically not operated with a chin rest. In order to evaluate the eye tracker's usefulness, its precision and accuracy should be tested in the most realistic conditions, so without a chin rest. As a consequence, the Eye Tribe tracker's recordings should be compared with those of an eye tracking system that also operates without a chin rest.

- The sampling rate of the EyeLink1000 $(1000 \mathrm{~Hz})$ is much higher than this of the Eye Tribe tracker (30 or $60 \mathrm{~Hz}$ ). The Eye Tribe tracker thus clearly belongs to the category of the low-speed systems and the EyeLink1000 eye tracker is a high-speed system. This brings along a number of important consequences (Holmqvist et al., 2011; Salvucci \& Goldberg, 2000): (1) Because of the short sampling interval, the high-speed eye trackers emit more IRlight to be able to detect the eyes' position; (2) Dalmaijer (2014) chose to apply the same event detection algorithms on both datasets (from the Eye Tribe versus the EyeLink1000), which would make the results comparable. With the high-speed device, more samples are included in one fixation compared to the low-speed device, allowing a more precise determine of the start and end of these fixations. Furthermore, when a higher number of gaze points are 
included in the definition of a fixation, its location can be determined more accurately.

- Another important difference is that the Eye Tribe tracker is a binocular system and the EyeLink1000 is a monocular system. Low-speed binocular systems only report the position of one eye, but the position of both eyes is used to increase the accuracy and precision of these reported locations (Holmqvist et al., 2011).

- In the proposed experiment, participants had to subsequently fixate nine points on the screen. The overall accuracy and precision of the recorded fixations were listed, but this was not related to the position of each point separately. This might provide vital insights regarding the accuracy and precision of the systems at different locations on the stimulus screen. This is especially interesting near the border of the screen, since this would be associated with the allowable tracking range of the eye tracker. The number of participants, five, is rather limited to be able to make strong statements. Because of this, no actual statistical tests were conducted and thus reported that could give insights in the level of significance of any differences.

Despite these shortcomings, we would like to stress the value of this evaluation as an initial starting point. We therefore like to take this paper as an opportunity to fine tune the evaluation process to be able to formulate more solid conclusions regarding the use of this low-cost eye tracker in scientific research.

In the next sections we present an overview of all elements and parameters in the design of the evaluation process (see also Figure 1), including justifications of the adaptations in relation to the evaluation procedures described by Dalmaijer (2014).

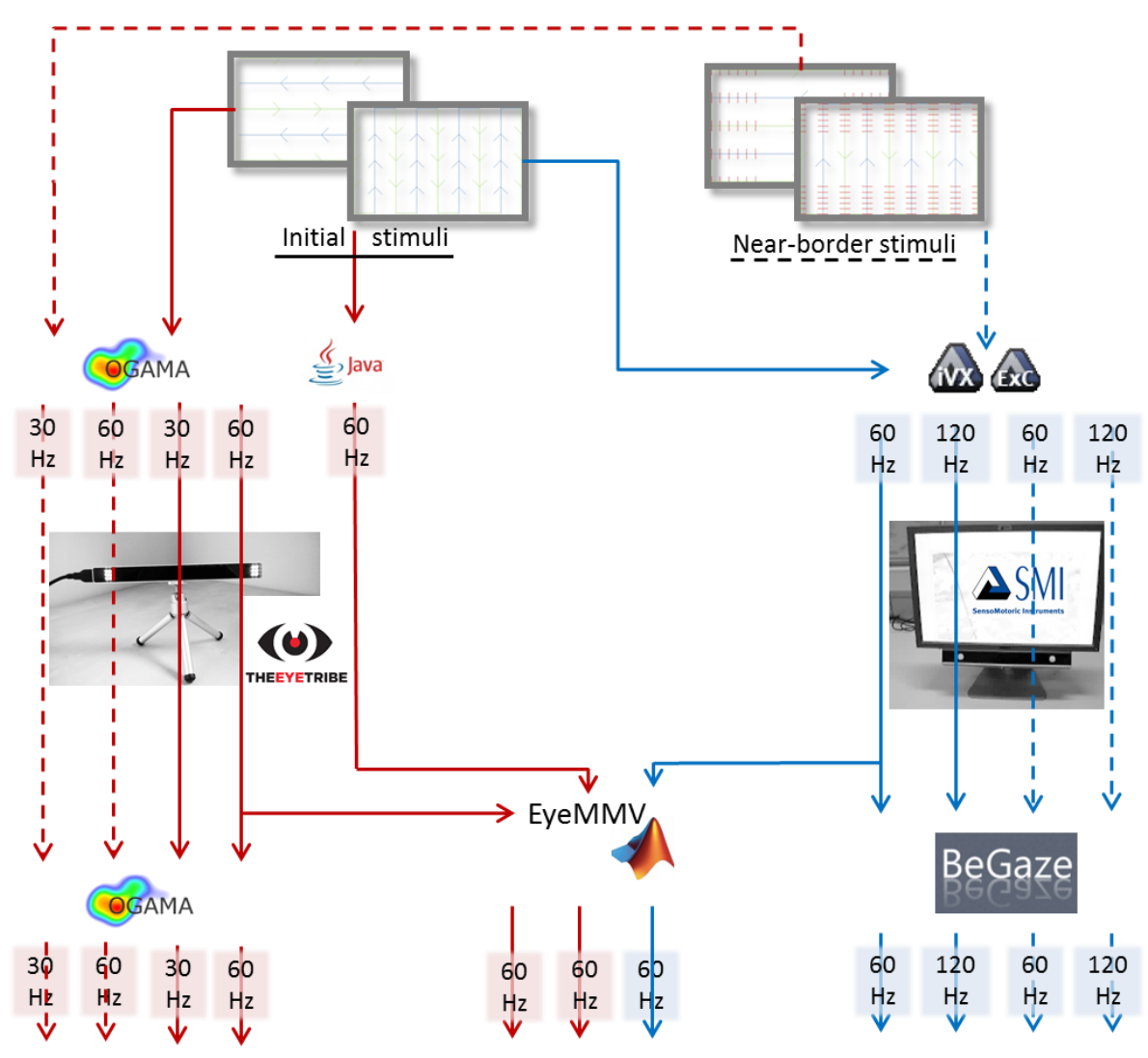

Figure 1: Overview of the experimental set-up and recordings. The solid lines represent trials in which the original stimuli are used; dashed lines correspond to trials in which the near-border stimulus is used. Red lines represent recordings with the Eye Tribe tracker; blue lines recordings with SMI RED. Furthermore, the sampling rate for each recording is mentioned, with an indication of different software packages that are used to record and process the raw data. 


\section{Apparatus}

The most important factor in the design of this study is the selection of the eye trackers to be evaluated. As we aim at extending the study of Dalmaijer (2014), the cheap and small-sized eye tracking system that is evaluated is the Eye Tribe tracker. The specifications of this device is listed in Table 1 (TheEyeTribe, 2015a). In order to be able to objectively evaluate this device, a comparable system has to be carefully selected. This system should have at least the following characteristics: can record data at $60 \mathrm{~Hz}$, remote system (without chin rest), binocular recordings, well-established system in scientific research. With this latter we mean that its characteristics should be of a high standard and that the system has already been extensively used in many scientific research fields. Two systems which perfectly fit these criteria are the SMI RED 250 eye tracker and the Tobii T60 (\& T120) eye
Ooms, K., Dupont, L., Lapon, L., \& Popelka, S. (2015) Evaluation of the accuracy and precision of a low-cost eye tracking device

tracker. For this study, the system of SMI was selected because it can record at $60 \mathrm{~Hz}$ (and $120 \mathrm{~Hz}$ ). This is adapted only by changing a parameter in the associated software that controls the eye tracking device: iViewX. Furthermore, the list of publications which report high qualitative studies based on an SMI RED is extensive (e.g. Bartels \& Marshall, 2012; Brychtova \& Coltekin, 2015; Cheng, Cristani, Stoppa, Bazzani, \& Murino, 2011; Dupont, Antrop, \& Van Eetvelde, 2015; Incoul, Ooms, \& De Maeyer, 2015; Ooms et al., 2015; Popelka \& Brychtova, 2013; Pretorius, Calitz, \& van Greunen, 2005; Sæther, Van Belle, Laeng, Brennen, \& Øvervoll, 2009; Strick, Holland, Van Baaren, \& Van Knippenberg, 2009). The specifications of the SMI RED 250 are listed in Table 1 (SMI, 2015). The recordings with the Eye Tribe tracker are visualized in red and grouped on the left side of Figure 1; those with the SMI RED 250 are positioned on the right and visualized in blue.

Table 1: Technical specifications of both eye tracking systems

\begin{tabular}{ccc}
\hline & $\begin{array}{c}\text { Eye Tribe tracker } \\
\text { (TheEyeTribe, 2015a) }\end{array}$ & $\begin{array}{c}\text { SMI RED 250 } \\
\text { (SMI, 2015) }\end{array}$ \\
\hline Eye tracking princi- & $\begin{array}{c}\text { Non-invasive, image based eye tracking: } \\
\text { pupil with corneal reflection }\end{array}$ & $\begin{array}{c}\text { Non-invasive, image based eye tracking: pupil } \\
\text { with corneal reflection }\end{array}$ \\
Sampling rate & $30 \mathrm{~Hz}$ or $60 \mathrm{~Hz}$ & $60 \mathrm{or} 120 \mathrm{~Hz}$ \\
Accuracy & $0.5^{\circ}-1^{\circ}$ & $0.4^{\circ}$ \\
Spatial resolution & $0.1^{\circ}(\mathrm{RMS})$ & $0.03^{\circ}$ \\
Latency & $<20 \mathrm{~ms}$ at $60 \mathrm{~Hz}$ & $<6 \mathrm{~ms}$ \\
Calibration & 9,12 or $16 \mathrm{points}$ & 2,5 or 9 points \\
Operating range & $45 \mathrm{~cm}-75 \mathrm{~cm}$ & $60-80 \mathrm{~cm}$ \\
Tracking area & $40 \mathrm{~cm} \times 30 \mathrm{~cm}$ at $65 \mathrm{~cm}$ distance $(30 \mathrm{~Hz})$ & $40 \mathrm{~cm} \mathrm{x} 20 \mathrm{~cm}$ at $70 \mathrm{~cm}$ distance \\
Gaze tracking range & Up to $24^{\prime \prime}$ & $40^{\circ}$ horizontal $\left(+/-20^{\circ}\right)$ \\
API/SDK & & $60^{\circ}$ vertical $\left(+20 /-40^{\circ}\right)$ \\
Data output & $\mathrm{C}++$, C\# and Java included & Free SDK/API, sample code \\
& Binocular gaze data & $($ e.g. Eprime, Matlab, C, C\#, Python) \\
\hline
\end{tabular}

The stimuli are for both systems presented on the same monitor, more specifically the 22 inch widescreen monitor to which the SMI RED 250 is attached. This monitor has a resolution of 1680 by 1050 pixels. Furthermore, this monitor is for both eye tracking systems located on the same position in the Eye Tracking Laboratory of the Department of Geography (Ghent University). This laboratory is fully equipped to conduct eye tracking studies and has, for example, shading curtains on the windows to block any interfering IR-light from the sun.

\section{Software for Experiment Set-up and Recordings}

For recording purposes, SMI Experiment Center was used to set-up and record the data for the SMI RED 250. The stimuli are uploaded in this system as separate images and the transition between the images is defined in this software. This package is connected with the SMI iViewX software which in turn communicates with the eye tracker itself. In iViewX, it is possible to change some of the eye tracker's settings, such as the sampling rate (with the options $60 \mathrm{~Hz}$ or $120 \mathrm{~Hz}$ ). 
The Eye Tribe tracker has two software packages that standardly accompany the device: the EyeTribe UI and the EyeTribe Server. The first one is a simple user interface which makes it possible to change some settings of the eye tracker, such as its sampling rate $30 \mathrm{~Hz}$ or $60 \mathrm{~Hz}$. Furthermore, the calibration procedure can be defined and initiated in this UI. The EyeTribe Server communicates with the device itself.

The data recorded with the Eye Tribe tracker has been collected in two ways. In one set of the tests, OGAMA was used. OGAMA (Open Gaze and Mouse Analyzer) is an open source software package, which allows to set-up, record and analyze eye tracking experiments using different eye tracking devices (Voßkühler et al., 2008). The Eye Tribe tracker is recently also supported by this software package, which means that OGAMA can communicate with the eye tracker and record the data it produces. In this set of experiments, the stimuli are thus also integrated in the set-up module in OGAMA as separate images including the definition of their transitions.

In a second set of tests, the data from the Eye Tribe tracker is recorded through a simple JAVA program which connects directly with the eye tracker's API, using the code provided by The Eye Tribe on https://github.com/EyeTribe/tet-java-client.git. The code for the applied JAVA-class can be found on https://gist.github.com/kristienooms/4c8df53f3800f46b19 58. The only thing this program does is accessing the recordings that the eye tracker produces and writing them into a text file. Consequently, there are no facilities in this JAVA-class to calibrate the eye tracker or define the setup of the study. The calibration of these experiments is done through the UI-module that comes along with the Eye Tribe tracker. Once the calibration has succeeded, the JAVA-class was initiated. The stimuli were presented using a PowerPoint presentation that was shown at full screen size on the monitor. The transition between the different images is thus defined as a transition between the slides of the presentation. An overview of these different recordings, related to each of the devices, is presented in Figure 1.

\section{Software for Data Analysis}

An important step in processing eye tracking data is the identification of eye tracking metrics, such as fixations and saccades. Since both eye tracking devices belong to the category of low-speed systems, dispersion- based algorithms need to be applied (Blignaut, 2009; Holmqvist et al., 2011; Salvucci \& Goldberg, 2000). These type of algorithms verify whether the raw eye tracking data falls within a certain radius (dispersion), taking into account a certain minimum fixation duration. Once a gaze point outside this radius is identified, this is considered as the start of a saccade and all previous gaze points are combined in one fixation. Nevertheless, different approaches exist to define and determine these fixations, even within the category of dispersion-based algorithms. As this has an influence on the (accuracy and precision of the) results, it is integrated into the study. Figure 1 gives an overview of the different software packages that are used to process the data.

Raw data recorded with the SMI RED 250 has to be consulted through the SMI BeGaze software package. With this package, the recorded raw data can be exported directly to an ASCII file that can be used for further processing in other software packages. On top of that, BeGaze itself provides functions to process and analyze the recorded data. This includes a dispersion-based algorithm to calculate fixations (among others), which requests the following parameters: minimal time (in ms) and dispersion (in pixels). The algorithm is explained in BeGaze's manual (SMI, 2012) and matches literally with the Dispersion-Threshold Identification (I-DT) algorithm as described by Salvucci and Goldberg (2000).

The algorithm is based on a moving window which contains a set of subsequent gaze points. Initially, the number of points contained in the window is defined by the parameter 'minimal time' and the sampling rate of the recordings. If the dispersion between all points is below the specified threshold, these gaze points belong to the same fixation. The calculation of the dispersion is based on the minimum and maximum values of $\mathrm{x}$ and $\mathrm{y}: \mathrm{D}=$ $(\max (x)-\min (x))+(\max (y)-\min (y))$. With each step, the next gaze point is added and the dispersion evaluated. If the dispersion above the threshold is found, all previous points define the fixation, which is positioned their centroid. Based on this we can conclude that the dispersion $\mathrm{D}$ does not correspond to the radius of a buffer around a central point, but relates more to the diameter hereof.

Nevertheless, there are no standard values defined for the threshold values in event detection algorithms. Blignaut et al. (2014) discussed the influence of varying values of the parameters 'minimal time' and 'dispersion' for the algorithm of Salvucci and Goldberg (2000). They 
argue that the accuracy of fixation detection is heavily dependent on a correct selection of these parameters' values. Commonly used threshold values are based on physiological characteristics, but also depend on the task at hand. However, high individual differences can be found in eye movement recordings (Blignaut et al., 2014; Duchowski, 2007; Holmqvist et al., 2011; Jacob \& Karn, 2003; Poole \& Ball, 2006; Popelka, 2014; Rayner, 1998).

In an earlier work, Blignaut (2009) found no optimum value for the dispersion parameter. However, dispersion thresholds (in visual angle, indicating a radius) from $0.7^{\circ}$ to $1.3^{\circ}$ were found produce acceptable indicator values. Taking into account these considerations, these parameters are selected for SMI's event detection algorithm: minimal time $=100 \mathrm{~ms}$ and maximal dispersion $=$ 80 pixels. The latter value corresponds to a visual angle of $2.2^{\circ}$. It must be noted here that this is not a radius but based on the description of the dispersion calculation.

OGAMA has a similar fixation detection function as BeGaze to process raw eye tracking data, which is described in the manual (Voßkühler et al., 2008). The algorithm is dispersion-based using a moving window, with a reference to Salvucci and Goldberg (2000). Nevertheless, in the manual of OGAMA it is mentioned that they consider a circular region around the (current) central position of the fixation that is calculated at that moment. They further specify "the radius of the acceptance circle" to evaluate the dispersion criteria.

Based on this description, it can be concluded that it is somewhat different from the algorithm used by BeGaze as the entered parameter for the dispersion D corresponds to the radius of the circle, with the average fixation as center point. Therefore, a value of 40 pixels (or visual angle of $1.1^{\circ}$ ) is used for this parameter. The parameter that defines the minimal time for a fixation is here expressed as 'number of samples'. In order to have a minimal time of $100 \mathrm{~ms}$, this parameter should be equal to: the sampling rate / 10. This corresponds to 3 samples for $30 \mathrm{~Hz}(3 \times 33,333 \ldots \mathrm{ms})$ and 6 samples for $60 \mathrm{~Hz}(6 \mathrm{x}$ $16,667 \mathrm{~ms})$.

The eye movements registered in JAVA were processed by an open source Matlab-based toolbox: EyeMMV. The algorithm used in this toolbox is described by Krassanakis et al. (2014). The spatial threshold is based on a circle (similar as in OGAMA), not a rectangle (as in BeGaze). In the article of Krassanakis et al.
(2014) the output of OGAMA and the EyeMMV tool are compared and found to be similar. Although this is not implemented in the current study, this algorithm can remove noise in the eye tracking data through the introduction of a second parameter, which is considered a weak element in the I-DT algorithms. The parameters used in this toolbox are: $100 \mathrm{~ms}$ as minimal fixation duration and 40 pixels $\left(1.1^{\circ}\right.$ visual angle) for both spatial thresholds (in order to simulate the algorithm used in OGAMA as good as possible). Because both threshold values are equal, no noise-removal is performed by the algorithm, which means it is reduced to a one-step process.

In order to be able to obtain insights in the influence of the event detection algorithms associated with the different systems an additional comparison is made. The raw data recorded with the SMI RED 250 and the Eye Tribe tracker is exported from BeGaze and OGAMA respectively. These datasets are processed again using the EyeMMV tool and compared with the results obtained with the other algorithms for that same dataset.

\section{Stimuli \& Task}

In order to evaluate the accuracy of an eye tracking system, participants have to subsequently fixate a number of fixed points distributed across the screen. However, care has to be taken in the design of these stimuli as this can influence the measures (Nyström et al., 2013). The stimuli constitute out of horizontal or vertical lines across the whole screen alternating depicted in green and blue. On these lines, arrows are visible in the same color as the line (see Figure 2).

Participants are asked to sequentially fixate the arrow and line endings (during 1-2 s) following the direction of the arrows. Using the crossings of lines as target points allows defining them very accurately. This time span is chosen because (1) it corresponds to that of Dalmaijer and (2) it is sufficiently long to detect the fixations even when considering a $30 \mathrm{~Hz}$ sampling rate.

In contrast to what was described by Dalmaijer, all fixation points are visible during the whole trial. This has some important consequences. Users are instructed to fixate the next target point after a certain time interval, but they have to decide for themselves when exactly do this. The targets of Dalmaijer are presented to activate bottom-up processes, whereas our approach is based on 
top-down processes or user driven activation (Wolfe, 1994, 2007).

Since all target points are visible during the whole trial, participants should be aware of their location even without paying explicit attention to them. First, the gist of a scene can be obtained quickly, even from a single fixation (Rayner, 2009). This is facilitated by the simple, but structured layout of the stimuli. The alternating line colors are an aid for the participants to grasp this structure upon first glance. Furthermore, using arrows the participants are guided in the right direction to select the next target point (Bertin, 1967). Second, covert attention always precedes a saccade (Wolfe, 1994, 2007). However, because participants are already familiar with the overall structure of the stimulus, it can be assumed that less covert attention need to be spent to calculate the eye movement to the next target point.

Since color and orientation are criteria that guide a participant's visual behavior, the directionality of the lines could have an important influence on the recorded gaze data (Wolfe, 1994). To compensate this issue, two stimuli are designed: one with horizontal lines and one with vertical lines (see Figure 2). Finally, all participants are introduced to the stimuli before the start of the actual tests, so they are already familiar with their layout.

Table 2: Coordinates of the fixation points on the horizontal and vertical stimulus

\begin{tabular}{lll}
\hline \multirow{2}{*}{ horizontal } & $\begin{array}{l}\text { X-coordinates } \\
\text { Y-coordinates }\end{array}$ & $0-420-840-1260-1680$ \\
& $0-263-252-788-1050$ \\
\hline \multirow{2}{*}{ vertical } & $X$-coordinates & $0-210-420-630-840-1050-1260-1470-1680$ \\
& $Y$-coordinates & $0-263-252-788-1050$ \\
\hline
\end{tabular}
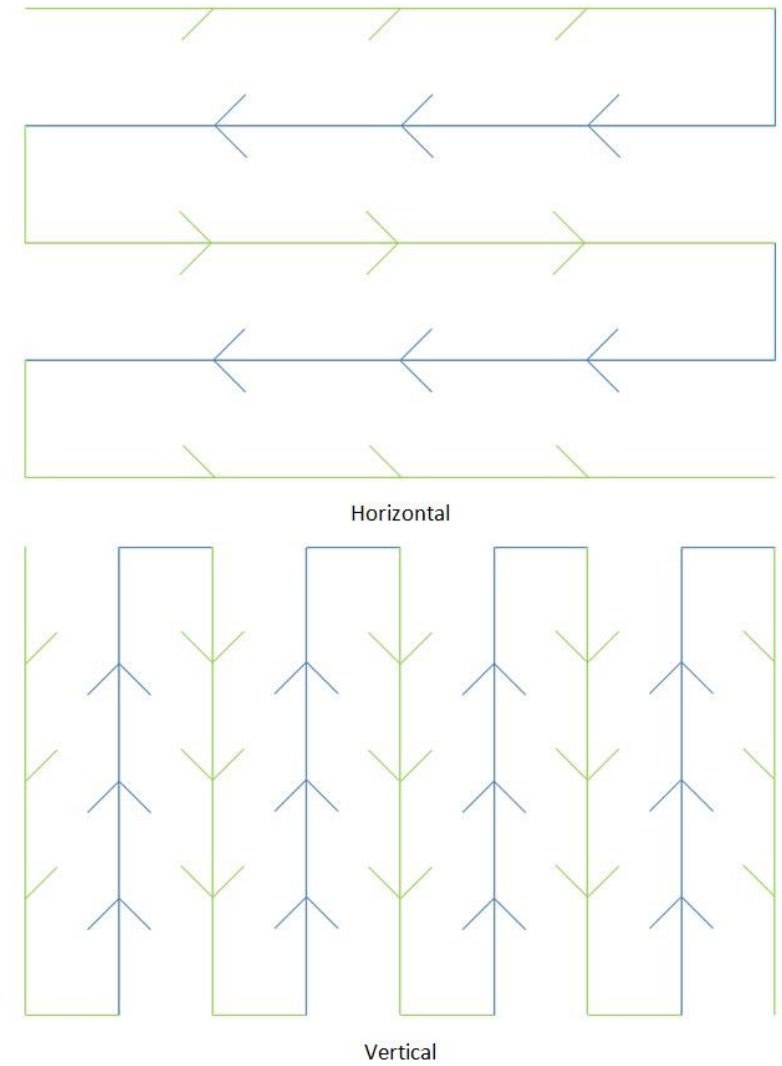

Figure 2: Original stimuli with homogeneous fixation points. Target points are arrow heads and line endings, which participants subsequently have to fixate. The stimulus with the horizontal lines is shown first; the one with vertical lines in the second trial
The aim of this experimental set-up is to have a controlled environment that still approaches real life experimental settings. The proposed task corresponds to, for example, reading (horizontal stimulus), following a path, or performing a visual search task. These are assignments which are often included in eye tracking research (e.g. Findlay \& Gilchrist, 1998; Incoul et al., 2015; Ooms et al., 2012; Rayner, 2009).

In the first stimulus (horizontal), five fixation points are depicted on each horizontal line and the image consists out of five lines. In the second stimulus (vertical) the fixation points are ordered in a vertical direction, with five fixation points on each line and nine lines in total. Consequently, much more fixation points are evaluated compared to the study of Dalmaijer (2014), including locations at the border of the stimulus screen. In order to calculate accuracy, the precise location of these fixation points has to be known (see Table 2). These are in screen coordinates (pixels) relative to the upper left corner of the screen.

During the initial tests with the Eye Tribe device (to determine its most optimal set-up, see further in the article), large deviations in the position of the recorded fixations were noticed at the (lower and right) border of the stimulus. Therefore, it was decided to create a second set of stimuli (see Figure 3) and conduct additional tests to determine where - near the border of the stimulus - the 
quality of the recorded fixation points deteriorates. In this second set of stimuli additional fixation points are added near the border: the portion of the line between the border and the first arrow point is divided in five equal portions by a small red dash depicted perpendicular on the line. Participants are asked to fixate on the intersection points of these red lines (and remaining arrow points). The two sets of stimuli are also included in Figure 1. The recordings with the initial stimuli are indicated by a solid line, recordings with the near-border stimuli are indicated with a dashed line.

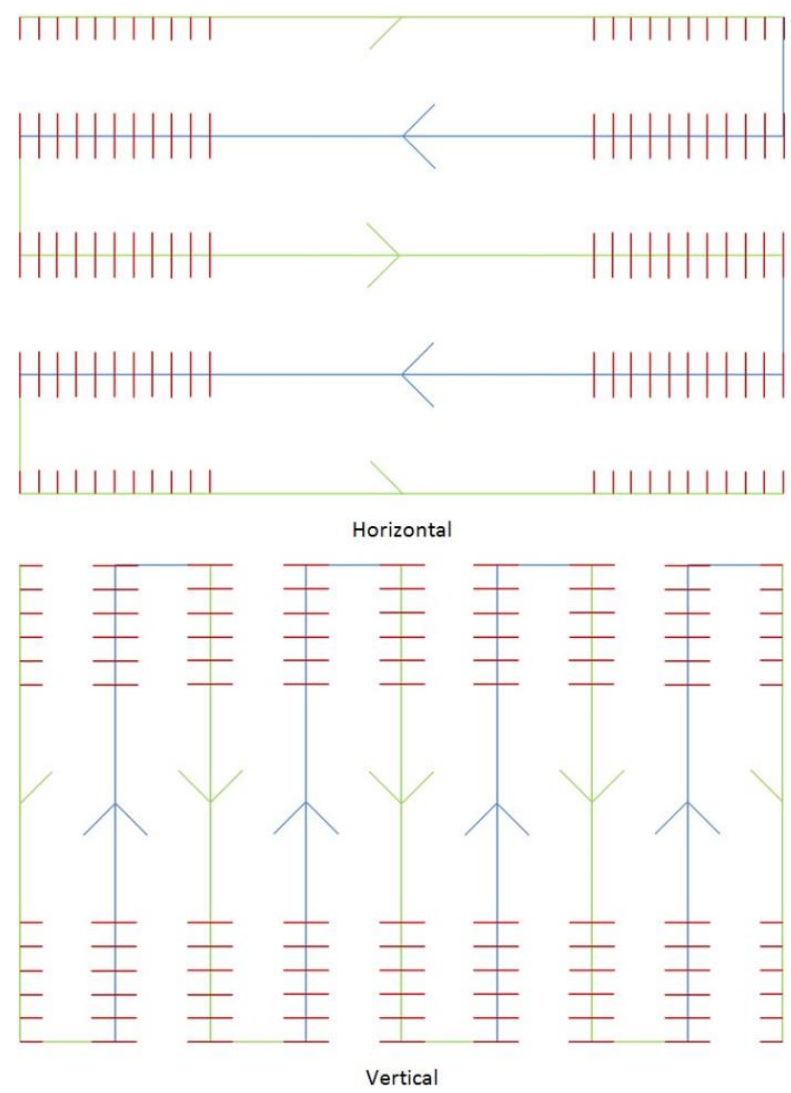

Figure 3: Stimuli for near-border testing. This is an extension on the initial stimuli with additional fixation points near the border, both for the horizontal and vertical lines. The additional target points are indicated by the intersection of the short red lines with the main lines.

\section{Participants}

In total 12 participants took part in this study. All were employees of the Department of Geography at Ghent University. In order to level out individual differences, the participants were involved in multiple tests with different settings: eye tracking device (SMI vs. Eye Tribe); sampling rate $(30 \mathrm{~Hz}$ vs. $60 \mathrm{~Hz}$ vs. $120 \mathrm{~Hz}$ ); re- cording software (OGAMA vs. JAVA). Since the participants are recorded multiple times, a minimal test group of ten subjects could be guaranteed for each trail. Because of the simplicity of the task (fixating subsequent points) the influence of a learning effect due to these repeated measures can be ruled out.

\section{Set-up Eye Trackers}

To register accurate results with an eye tracking device, its set-up is of utmost importance. The SMI RED 250 is attached in a fixed position to the monitor which is also provided by SMI. Only the distance between the participant and the monitor can vary, which should be between $60 \mathrm{~cm}$ and $80 \mathrm{~cm}$ as stated by the device's specifications. In order to deal with different participant heights, the angle of the monitor can be adjusted.

The Eye Tribe tracker has much more freedom regarding how it can be positioned. It comes along with a small tripod on which it can be mounted. Once it is attached to this tripod the angle of the device can still be adjusted (in all directions). Of course, to be able to record accurate data, the eye tracker should be placed perfectly horizontal and in the middle of the screen (in the horizontal direction). Besides these obvious settings, there are still a number of other settings that can vary:

- Horizontal distance participant - screen;

- Horizontal distance participant - eye tracker;

- (Horizontal distance eye tracker - screen);

- Height screen;

- Height eye tracker;

- Height participant;

- Vertical angle eye tracker.

On the website of The Eye Tribe, it is specified how the device should be positioned (TheEyeTribe, 2015b). They state that the eye tracker (on its tripod) should be placed on a flat surface, centered below the monitor (24 inches maximum). The distance between the monitor and participant should be $45-75 \mathrm{~cm}$ and the angle of the Eye Tribe tracker should be adjusted so it is directed towards the participant's face. The EyeTribe UI shows the participants eyes when they are detected by the device, and indicates whether they are positioned correctly (using a green background color). 


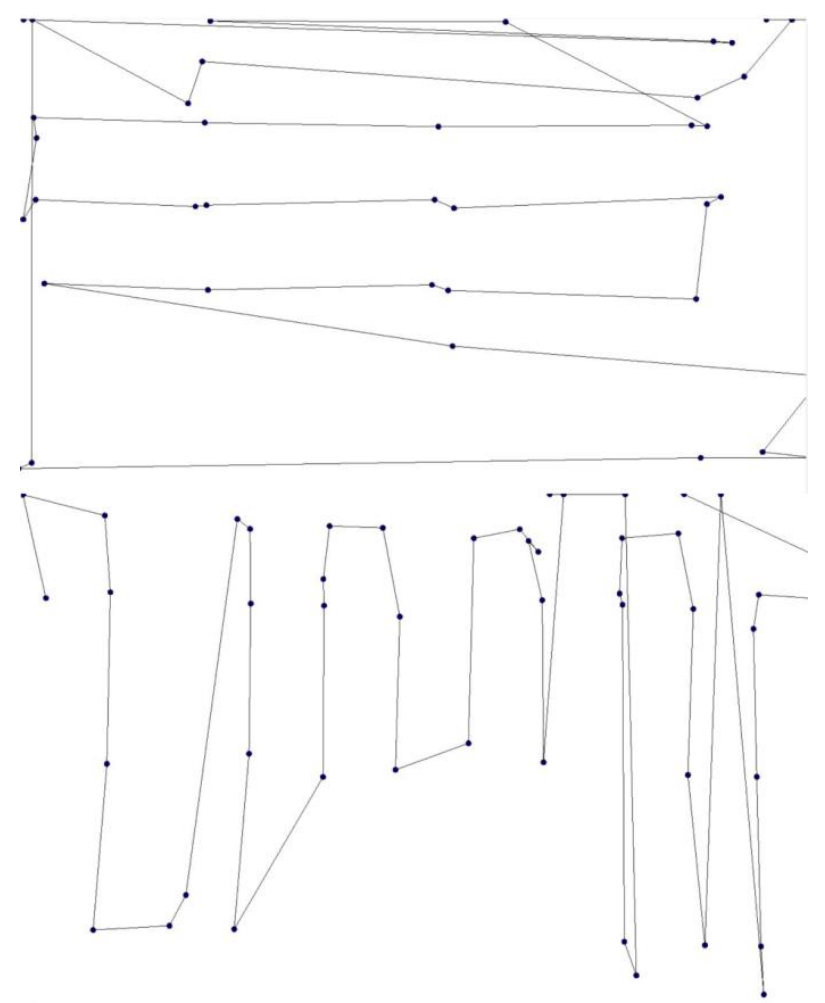

Figure 4: Recorded scanpaths during a non-optimal set-up. First image: poor recordings on the horizontal stimulus; second image: poor recordings on the vertical stimulus. Both lack fixation on multiple target points.

The set-up of the Eye Tribe tracker was evaluated by the authors in over a 100 tests in which the parameters mentioned before were varied staying within the prescriptions of The Eye Tribe (TheEyeTribe, 2015b). These initial evaluations were carried out on the 22 inch monitor that was used during the main experiment. The stimuli presented in Figure 2 were used to (visually) evaluate the recordings. During these tests was found that the eye tracker can be positioned within these guidelines, resulting in a green color for the right positioning but that (1) the calibration could not be executed with a sufficient quality or (2) the calibration could be performed with sufficient quality but the recorded data showed large deviations from the fixation points. This is illustrated in Figure 4. In most cases, the recordings at the border of the screen showed strikingly large deviations signaling a problem regarding the eye tracker's tracking range. This is also related to the height and distance (and consequently the vertical angle) of the eye tracker relative to the participant.
Ooms, K., Dupont, L., Lapon, L., \& Popelka, S. (2015) Evaluation of the accuracy and precision of a low-cost eye tracking device

Based on the variations in the $>100$ test described above, a set of best practices guidelines could be derived and these were further verified on a 20 inch $(1980 \times 1080$ pixels) monitor and 18.5 inch (1280 x 1024 pixels) monitor. These guidelines are described below and illustrated in Figure 5:

- Participants should sit up straight;

- Height of the stimulus screen: participant's eyes should be positioned halfway on the screen when looking straight ahead;

- Distance participant - stimulus screen: This should be more than $45 \mathrm{~cm}$ and a preferable distance of 60 $\mathrm{cm}$ was found. Positioning the screen closer allows participants to distinguish more details. However this introduces a wider visual angle for the participant to see the screen and the eye tracker might lose track of the participant's eyes when (s)he is looking at the border of the screen;

- Distance participant - eye tracker: this distance should be sufficiently close to ensure an accurate calibration, but not too close as this again enlarges the angle for the eye tracker to detect the participant's eyes. A preferable distance of $30 \mathrm{~cm}$ to $45 \mathrm{~cm}$ was observed during the evaluations;

- Height eye tracker: the top side of the eye tracker should be aligned with the underside of the stimulus screen.

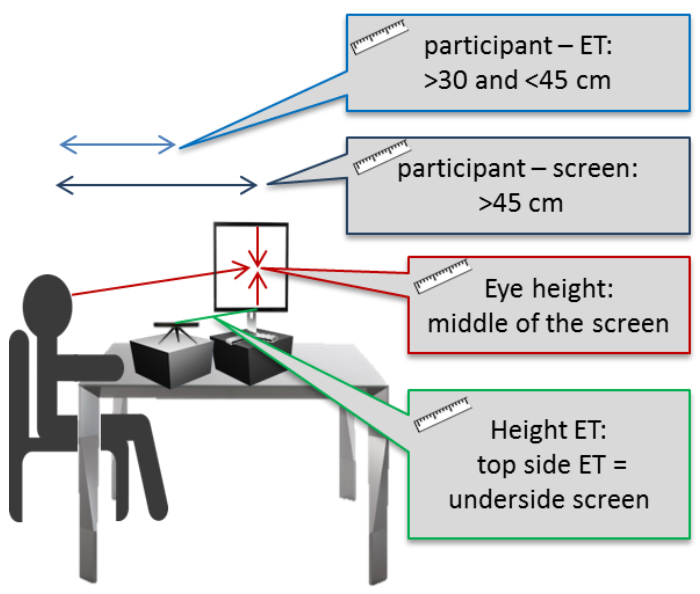

Figure 5: Best practices to set-up the Eye Tribe device 


\section{Results}

In contrast to Dalmaijer (2014), accuracy and precision will be reported for each fixation point separately. This huge amount of information will be visualized using a color coded graphics, supplemented with icons. The legends associated with the color code and icons are explained in detail in the separate sections below.

\section{Evaluation of Accuracy and Precision}

For each fixation point the distance (in pixels) is calculated between the real location of this point and the recorded fixation on this point. The average values and related standard deviations are visualized in Figure 6. Each separate image in this figure corresponds to the stimulus screen and each cell in such a graphic corresponds to the fixation point at the corresponding location on the stimulus screen. The colors in the grid give an indication of the size of the offset and the icons (pie charts) visualize the size of the standard deviation (or precision) at that location on the screen. The numbers in the cells represent the average offset value for that fixation point. These graphics are structured in a table to be able to compare the obtained results in structured way. Besides the different eye tracking devices, special attention is paid to recordings with differing sampling rates and the applications of different fixation detection algorithms. The classification (or legend) is based on the reported average accuracy of the Eye Tribe tracker, which is $0.5^{\circ}-1.0^{\circ}$. The distance between the stimulus screen and the participants was $60 \mathrm{~cm}$, so the (maximal) visual angle of $1.0^{\circ}$ corresponds to about 40 pixels on the screen.

\section{Initial Distribution of Fixation Points}

With the initial stimuli, the fixation points are distributed homogeneously across the stimulus screen. The first thing that can be noticed is the overall high offset values for the Eye Tribe tracker when using a sampling rate of $30 \mathrm{~Hz}$. This sampling rate is actually rather low to be used in scientific experiments on a static screen, but it was included to be able to compare its results to the ones obtained when using a sampling rate of $60 \mathrm{~Hz}$. It is immediately clear that the offset values are much lower
Ooms, K., Dupont, L., Lapon, L., \& Popelka, S. (2015) Evaluation of the accuracy and precision of a low-cost eye tracking device

using a sampling rate of $60 \mathrm{~Hz}$. However, the locations of the fixations at the bottom and right edge are not recorded near the actual fixation point location. These locations are also associated with a high value for the standard deviation, which is linked to a low precision.

The same (raw) data that was recorded at $60 \mathrm{~Hz}$ in OGAMA, is also exported and processed to fixations using the EyeMMV script in Matlab (Krassanakis et al., 2014). This procedure makes it possible to investigate the influence of the fixation detection algorithm on the accuracy and precision of the results. So although the same raw data is used as input, differences in the results are still observable. These are the result of assigning individual gaze points to a different fixation, causing a change in the fixations' locations (average of all gaze positions it includes).

When comparing the previous results with the average offsets that are measured when operating the Eye Tribe device trough the JAVA program, the values at the lower and right edge are not deviating as much. Nevertheless, the overall average offset values seem to be a bit higher in the other parts of the screen. The precision of these recorded locations is mostly situated within the boundaries of the best class.

The offset values related to the SMI RED recordings agree most with those of the Eye Tribe device when using the JAVA program: somewhat higher values in the main part of the screen, but no extremely large deviations on the lower and right border. The eye movements are normally recorded and processed in SMI Experiment Suite 360 (which includes Experiment Center and BeGaze). However, besides this regular process the raw data was exported from BeGaze and processed to fixations using the EyeMMV script in Matlab (see last row of Figure 6). Also in this case, due to the re-assignment of gaze positions to fixations, an influence of the different fixation detection algorithm (details are discussed before) is noticeable in the results. Finally, the SMI RED allows to record eye movements with a higher sampling rate: 120 $\mathrm{Hz}$. Nevertheless, the average offset values (and related standard deviations) are not smaller when using this higher sampling rate. 


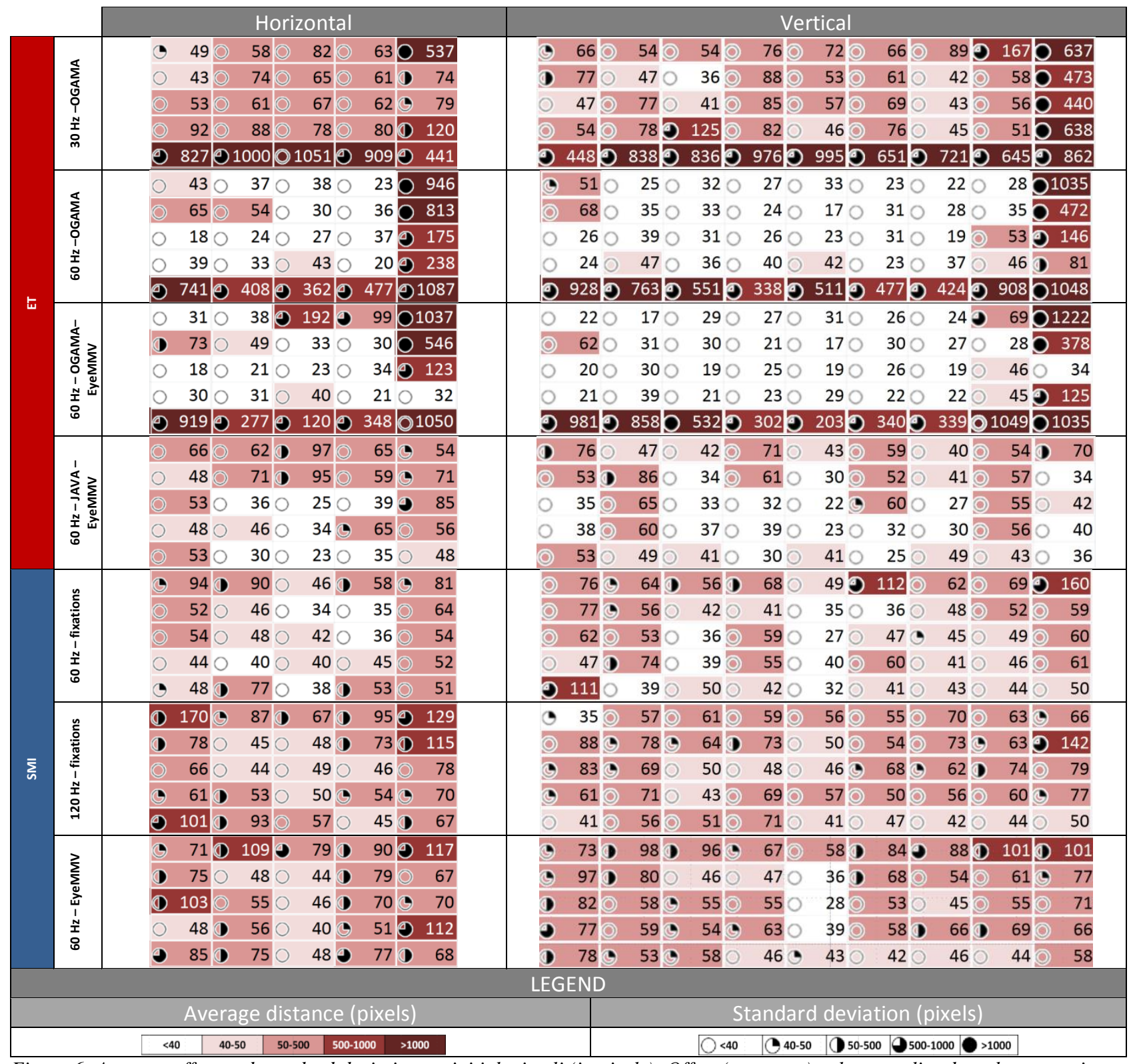

Figure 6: Average offset and standard deviation on initial stimuli (in pixels). Offset (accuracy) values are listed on the approximate corresponding positon on the screen in a tabular layout. The color of the cells is linked to the offset value, categorized in five steps. The standard deviation (precision) is visualized using a pie chart that is added to the corresponding cell, also using the same five categories.

\section{Near-Border Testing}

Since the Eye Tribe tracker shows extremely large deviations in the offset values near the lower and right border of the screen, a second set of stimuli is evaluated. These include a denser number of fixation points near the border of the stimulus screen (see Figure 3 for the stimuli). The resulting values for the offset and standard devia- tion are visualized in Figure 7. The eye movements are, respectively, recorded and analyzed with OGAMA and SMI Experiment Suite only. Nevertheless the different sampling rates are maintained in the evaluation procedure. The data indicate that the extreme deviations only occur at the outer limit of the stimulus screen, so not on the fixation points that are located only a little bit more inwards. 


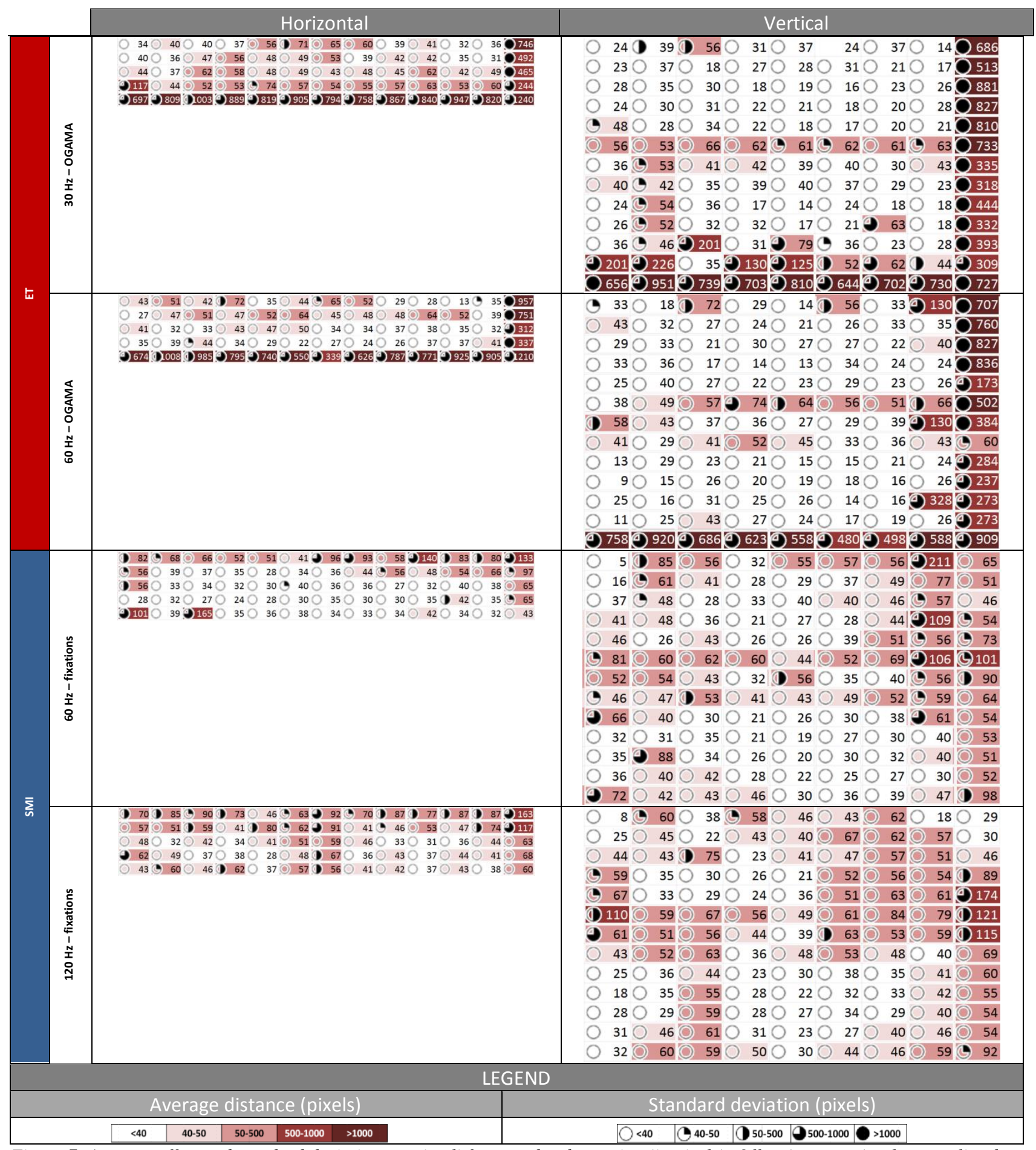

Figure 7: Average offset and standard deviation on stimuli for near-border testing (in pixels). Offset (accuracy) values are listed on the approximate corresponding positon on the screen in a tabular layout. The color of the cells corresponds to the offset value, categorized in five steps. The standard deviation (precision) is visualized using a pie chart that is added to the corresponding cell, also using the same five categories. 
Journal of Eye Movement Research $8(1): 5,1-24$

\section{Evaluation of Directionality}

The offset values give a good indication of the average distance between the fixation point and the recorded fixation, but it does not provide any information regarding the main direction of the deviations. Therefore, Figure 8 visualizes the average distances in $\mathrm{X}$ and $\mathrm{Y}$ between the fixation point and the recorded fixations, taking the direction of the deviation into account: a positive deviation is to the right in $\mathrm{X}$ and to the bottom in $\mathrm{Y}$; a negative is to the left in $\mathrm{X}$ and towards the top in Y. The point of origin for these measurements is located in the upper left
Ooms, K., Dupont, L., Lapon, L., \& Popelka, S. (2015) Evaluation of the accuracy and precision of a low-cost eye tracking device

corner of the stimulus screen. In order to stay within the page limits of this journal, only the basic $60 \mathrm{~Hz}$ tests on the Eye Tribe tracker and the SMI RED are reported.

The presented values are much smaller than the offset values visualized in Figure 6, which is an effect of (1) considering only two perpendicular axis and (2) the plus and minus signs which neutralize each other when calculating the average. Nevertheless, the extreme values near the lower and right edge become apparent again for the Eye Tribe tracker.

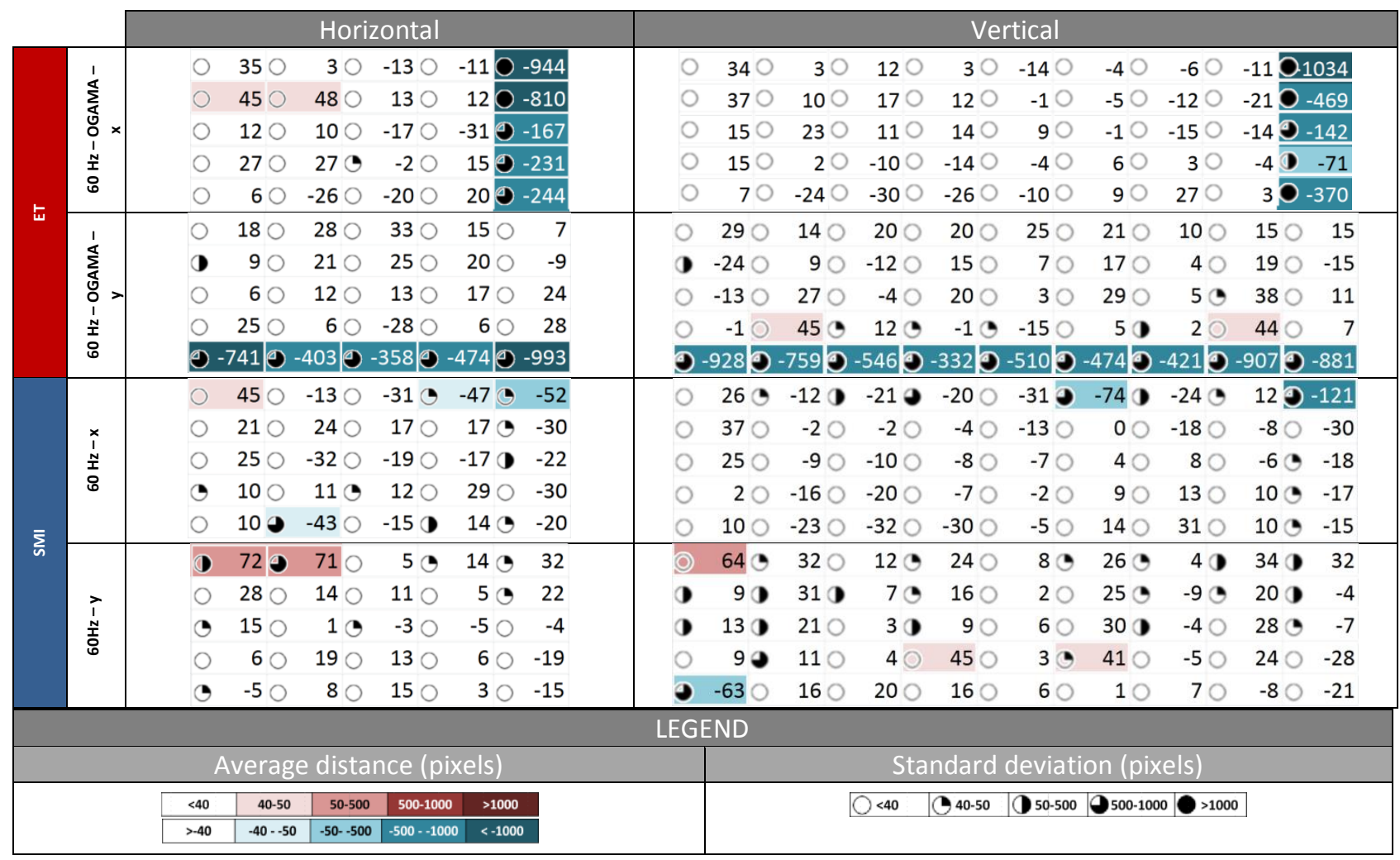

Figure 8: Average offset and standard deviation in $X$ and $Y$ on initial stimuli (in pixels). Offset (accuracy) values are listed on the approximate corresponding positon on the screen in a tabular layout. The color of the cells corresponds to the offset value, distinguishing between positive (red) and negative (blue) values. Similar as with the previous figures, there are five steps for each color. The standard deviation (precision) is visualized using a pie chart that is added to the corresponding cell, also using the same five categories.

\section{Statistical Comparison}

Besides the visual inspections on the average offsets and standard deviations for the different fixations points, a more solid approach is needed to be able to derive conclusions. In contrast to Dalmaijer (2014), a statistical comparison of the offset values is presented here. These comparisons not only consider the absolute distances but also the directional values in $\mathrm{X}$ and $\mathrm{Y}$. Since the recordings do not have a normal distribution, a non-parametric approach is necessary. The results - mean (M), medium (Med), standard deviation (SD), and outcome of the Median and Mann-Whitney U test - are listed in Table 3. 


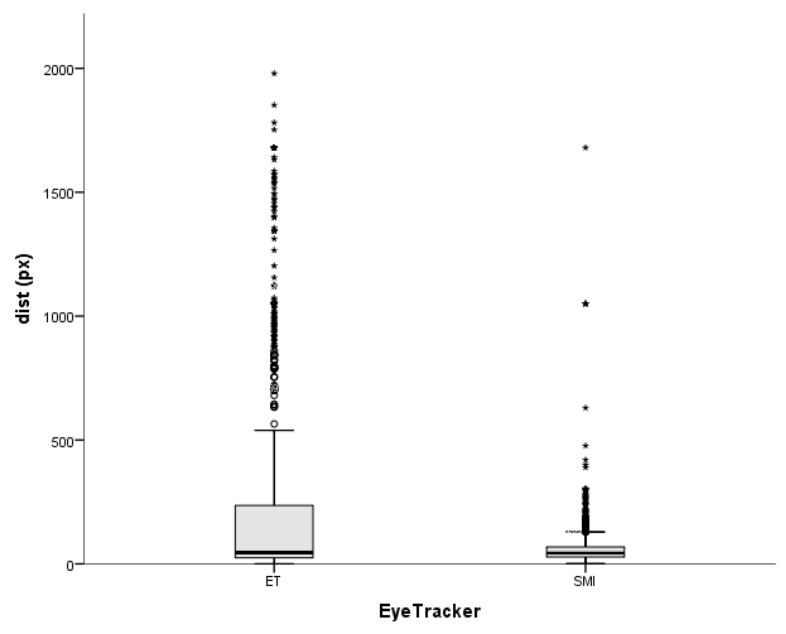

Figure 9: Boxplots of the registered offset values for both eye tracking devices. Offset values illustrate the accuracy of the fixation location with the Eye Tribe tracker (ET, left) and the SMI RED 250 (SMI, right). The distribution of these values is linked to precision of the recordings.

The Median test only indicates a significant difference in the offset values in the Y-direction. The results of the
Mann-Whitney $U$ test indicate that the accuracy of the eye movements recorded with the SMI RED are significantly better than those recorded with the Eye Tribe tracker and this holds true for all variables. Furthermore, the boxplot in Figure 9 gives an indication on the higher precision of the SMI RED recordings.

Since lowest accuracy for the Eye Tribe tracker was obtained at the border of the stimuli, a second set of tests were executed which eliminate all border-measurements. The results are listed in the lower half of Table 3. Strikingly, still many significant differences are found. However, in this case the best results are obtained with the Eye Tribe tracker, which now shows much lower values for the mean, median and standard deviation in the different distances. Opposite to the initial tests, the median values are significantly different for dist and dist $X$, but not for distY. The same results are obtained from the Mann-Whitney U tests.

Table 3: Statistical comparison of the registered offset values (with and without border values)

\begin{tabular}{|c|c|c|c|c|c|c|c|c|c|}
\hline \multirow[b]{2}{*}{ All values: } & \multicolumn{3}{|c|}{ dist } & \multicolumn{3}{|c|}{ distX } & \multicolumn{3}{|c|}{ distY } \\
\hline & $\mathrm{M}$ & Med & SD & $\mathrm{M}$ & Med & SD & $\mathrm{M}$ & Med & SD \\
\hline ET & 274 & 46 & 440 & -97 & -5 & 343 & -152 & $-0,69$ & 344 \\
\hline SMI & 57 & 44 & 69 & -10 & -6 & 68 & 12 & 11 & 56 \\
\hline Med-test & & .280 & & & .594 & & & .000 & \\
\hline Mann-Whitney U test & & .000 & & & .030 & & & .000 & \\
\hline No border values: & $\mathrm{M}$ & Med & SD & $\mathrm{M}$ & Med & SD & $\mathrm{M}$ & Med & SD \\
\hline ET & 34 & 28 & 25 & 2 & 1 & 24 & 12 & 12 & 32 \\
\hline SMI & 45 & 38 & 34 & -1 & -3 & 31 & 12 & 13 & 46 \\
\hline Med-test & & .000 & & & .004 & & & .653 & \\
\hline Mann-Whitney U test & & .000 & & & .004 & & & .348 & \\
\hline
\end{tabular}




\section{Sampling Rate}

Another important element regarding the reliability of an eye tracking device is the precision of the sampling rate (the temporal precision). In the previous test, two eye tracking devices are evaluated which both recorded eye movements with a sampling rate of $60 \mathrm{~Hz}$. In theory this would mean that one recording takes place every 16.667 ms. Table 4 shows the percentages of the measurements which took place after a certain time interval. The SMI RED has more than $99.8 \%$ of its recordings within the time interval (16.5 ms - $17.5 \mathrm{~ms})$, corresponding to a sampling rate of $60 \mathrm{~Hz}$. A much lower value is found for the Eye Tribe tracker: only about $45.1 \%$. A large part of the recordings from this device occurs after a shorter time interval (15.5 ms - $16.5 \mathrm{~ms}$ ). However, these recordings still count up to only $87.1 \%$ of the recordings, which means that all other registrations occurred after time intervals with a much larger deviation from the theoretical $60 \mathrm{~Hz}$.

Dalmaijer (2014) reported that $99.08 \%$ of his Eye Tribe recordings were performed with a sampling rate within the time interval $(14.67 \mathrm{~ms}-18.67 \mathrm{~ms})$. Since this time interval is somewhat larger than the one mentioned considered in previous paragraph, the value of $87.1 \%$ should probably be increased somewhat (to around $90 \%$, considering the $8.3 \%$ of measurements included in the next category). Still, a rather large difference - of more than $9 \%$ - can be noticed here. This difference could be explained by a difference in the detection of invalid/missing values.

Table 4: Recorded time intervals between samples at $60 \mathrm{~Hz}$

\begin{tabular}{ccc}
\hline $\begin{array}{c}\text { Time interval } \\
(\mathbf{m s})\end{array}$ & $\begin{array}{c}\text { SMI 60Hz } \\
(\%)\end{array}$ & $\begin{array}{c}\text { ET 60 Hz } \\
(\%)\end{array}$ \\
\hline$[\mathbf{1 4 . 5 - 1 5 . 5 )}$ & 0.012 & 0.010 \\
{$[\mathbf{1 5 . 5 - 1 6 . 5})$} & 0.042 & 42.008 \\
{$[\mathbf{1 6 . 5 - 1 7 . 5})$} & 99.830 & 45.107 \\
{$[\mathbf{1 7 . 5 - 2 0 . 5})$} & 0.015 & 8.259 \\
{$[\mathbf{2 0 . 5 - 3 0 . 5 )}$} & 0 & 0 \\
{$[\mathbf{3 0 . 5 - 4 0 . 5 )}$} & 0 & 4.532 \\
$\geq \mathbf{4 0 . 5}$ & 0.101 & 0.084 \\
\hline
\end{tabular}

Dalmaijer (2014) reported $6.74 \%$ missing values, but also a much lower percentage of recordings within in the
Ooms, K., Dupont, L., Lapon, L., \& Popelka, S. (2015) Evaluation of the accuracy and precision of a low-cost eye tracking device

categories with higher time intervals compared to our study (> $18.67 \mathrm{~ms}: 0.92 \%$ vs. > $20.5 \mathrm{~ms}: 4.62 \%$, respectively). However, no explanation is given regarding the detection of invalid/missing values when processing the data so it is impossible to reproduce this. In our study the data was processed by three types of software (SMI BeGaze, OGAMA, and JAVA). When evaluating the sampling rate for the ET the recordings from JAVA were used to avoid any (unforeseen) filtering procedures in the software.

\section{Discussion}

Using the experimental set-up described above, the accuracy and precision of eye tracking devices were evaluated taking into account a number of criteria: eye tracking device, sampling rate, fixation detection algorithm, and fixation point location. From the results it can be concluded that the accuracy and precision of the recordings registered with the low-cost Eye Tribe tracker cannot only be related to the characteristics of the device itself. There are many influencing factors which could have a negative impact on the quality (accuracy and precision) of the recordings, which holds also true for the more expensive SMI RED 250.

The Eye Tribe device has the advantage that it is easily transportable and can be set-up very quickly: place it on its tripod and in between the screen and the participant. However, care has to be taken in the set-up of the device as in this step already there are many parameters that could make the recordings useless: position relative to the participant, position relative to the screen, lighting conditions in the room (e.g. sunlight), etc. Especially because of its transportability, this - time consuming process has to be repeated with every set-up. It is good practice to have some stimuli (similar to the ones used in this experiment) to evaluate the quality of the device's set-up.

The results of the recordings at $30 \mathrm{~Hz}$ are the least accurate, although the precision (standard deviation) is still situated in the best category. The minimum number of samples - or gaze position - to be included in a fixation is only three, and a time interval of $33,333 \mathrm{~ms}$ exists between the subsequent registered gaze position. Both of these elements contribute to the fact that more accurate positions can be determined at $60 \mathrm{~Hz}$, indicating that the $30 \mathrm{~Hz}$ alternative is less suited to use in scientific exper- 
iments. However, a less pronounced difference is seen when comparing the accuracy and precision of the outcomes recorded with the SMI RED at $60 \mathrm{~Hz}$ and $120 \mathrm{~Hz}$ respectively. At first sight the results at $120 \mathrm{~Hz}$ seem to be of a lesser quality, but the differences are actually rather small; most of them are in the order of 10 pixels offset.

Although the different fixation detection algorithms are highly - but not exactly - similar, minor influences in the results can be seen when processing the same raw data with a different algorithm. This both holds true for the OGAMA-EyeMMV and the BeGaze-EyeMMV comparison. The observed differences are a consequence of assigning the gaze positions to different clusters which define the fixations. This causes minor shifts in the locations of the fixations and thus the related offset values.

The main aim of this experiment is to determine the accuracy and precision of the low-cost Eye Tribe tracker in different experimental set-ups and this in comparison to the results of the well-established and more expensive SMI RED 250. Two things can be noted here. First, the Eye Tribe tracker's recordings show high deviations at the edge of the screen; both in terms of offset and standard deviation. These deviations are most apparent at the lower and right edge. When studying these deviations more closely it became clear that these might relate to occasions where the eye tracker loses contact with the eye (corneal reflection and pupil center). At the lower edge this resulted in (near) zero recordings for the $y$ value and at the right edge in (near) zero values for the $\mathrm{x}$ value. Nevertheless, since the origin of the associated coordinate system is situated in the upper left corner, the $\mathrm{x}$ - and $\mathrm{y}$-values of, respectively, the left and upper edge of the screen correspond to zero. Although no large deviations are recorded here, the same issue might be occurring at these locations. Nevertheless, the more detailed tests near the border of the screen indicate that this problem only occurs at the outer edge of the stimulus screen, not when the fixation points are located a little bit more inward.

On the contrary, these issues near the border do not seem to occur when recording data using the JAVA program instead of using OGAMA. However, the overall offset values are on the higher side. We assume that this latter issue is related to the quality of the calibration process, which can have a large (negative) influence on the theoretical accuracy values $\left(0.5^{\circ}-1.0^{\circ}\right.$ for the Eye Tribe device). In OGAMA, calibrations up to 'moderate' were accepted if the distribution quality of the calibration was evenly distributed across the calibration points. Before starting the JAVA program, Eye Tribe's user interface (UI) was used to start the calibration. However, the feedback on the quality of the calibration process is less detailed in this case, with no indication of the validation values (as in SMI Experiment Center) or its distribution across the calibration points. Therefore, it is difficult to estimate the real quality of the calibration using this tool.

The above mentioned issues do not occur when analyzing the recordings from the SMI RED. Nevertheless, the overall offset values seem to be somewhat higher. At first sight, this is especially striking since the theoretical visual accuracy should be better $\left(<0.4^{\circ}\right)$. As mentioned before, the quality of the calibration process could have an influence on this. During the calibration process, validation values up to around $0.5^{\circ}$ (in $\mathrm{X}$ and $\mathrm{Y}$ ) for the SMI RED were allowed, which means that the theoretical values for accuracy $\left(<0.4^{\circ}\right)$ would not be reached. However, the values obtained in these calibration processes reflect what can be expected when conducting a normal scientific experiment (both regarding the Eye Tribe device and the SMI RED). Nevertheless, the precision of the SMI recordings is equally lower, indicated by the higher values for the standard deviations.

In the statistical comparison, the offset values (absolute distances and directional distances in $\mathrm{X}$ and $\mathrm{Y}$ ) are evaluated for the two devices operating at $60 \mathrm{~Hz}$. The outcomes show a significantly better result for the SMI RED for all parameters. Nevertheless, the extreme deviations near the border have a strong negative influence on the overall result of the recordings from Eye Tribe. When only considering the non-border measurements, a significant difference is still reported, but now in favor of the Eye Tribe tracker.

A second element was the evaluation of the sampling ratio of both devices. In this case, a much better performance - which can be translated in reliability - is found for the SMI RED having a stable time interval between subsequent measurements. Many small and some large deviations on this were found in the raw recordings of the Eye Tribe tracker. 


\section{Conclusion}

To conclude, the Eye Tribe tracker is a valuable instrument for academic research; when used correctly, the accuracy and precision of its outcomes are comparable to those of comparable well-established eye tracking devices, such as the SMI RED 250. However, the main pitfall here is the note 'when used correctly', because there are many factors which can render the recordings useless. These factors - such as the set-up, the software to calibrate, record and process the data - are already carefully evaluated by the producers of the (more expensive) wellestablished eye trackers and integrated in a packages that come along when ordering these eye trackers: laptop, software packages to set-up and conduct the experiments, stimulus screen, possibilities to attach the eye tracker to this stimulus screen (so it is automatically correctly positioned), etc. The low-cost eye trackers do not have this type of coverage around it, making them more prone to errors in their usage resulting in outcomes with an inferior quality.

\section{References}

Akinlofa, O. R., Holt, P. O. B., \& Elyan, E. (2014). The cognitive benefits of dynamic representations in the acquisition of spatial navigation skills. Computers in Human Behavior, 30, 238-248.

Bartels, M., \& Marshall, S. P. (2012). Measuring cognitive workload across different eye tracking hardware platforms. Paper presented at the Proceedings of the symposium on eye tracking research and applications.

Berces, A., \& Török, Z. G. (2013). A home-made 10 bucks eye tracking system. Paper presented at the Workshop on Eye Tracking: Why, When, and How?, Dresden.

Bertin, J. (1967). La sémiologie graphique. Paris: Mouton-Gauthier-Villars.

Blignaut, P. (2009). Fixation identification: The optimum threshold for a dispersion algorithm. Attention, Perception, \& Psychophysics, 71(4), 881-895.

Blignaut, P., Holmqvist, K., Nyström, M., \& Dewhurst, R. (2014). Improving the accuracy of videobased eye tracking in real time through postcalibration regression Current Trends in Eye Tracking Research (pp. 77-100): Springer.

Borji, A., \& Itti, L. (2014). Defending Yarbus: Eye movements reveal observers' task. Journal of Vision, 14(3), 29.
Ooms, K., Dupont, L., Lapon, L., \& Popelka, S. (2015)

Evaluation of the accuracy and precision of a low-cost eye tracking device

Brychtova, A., \& Coltekin, A. (2015). Discriminating classes of sequential and qualitative colour schemes. International Journal of Cartography, $1(1), 62-78$.

Buswell, G. T. (1935). How people look at pictures University of Chicago Press (pp. 142-144). Chicago University of Chicago Press.

Cheng, D. S., Cristani, M., Stoppa, M., Bazzani, L., \& Murino, V. (2011). Custom Pictorial Structures for Re-identification. Paper presented at the BMVC.

Dalmaijer, E. (2014). Is the low-cost EyeTribe eye tracker any good for research? (2167-9843). Retrieved from

Dalmaijer, E., Mathôt, S., \& Van der Stigchel, S. (2014). PyGaze: An open-source, cross-platform toolbox for minimal-effort programming of eyetracking experiments. Behavior research methods, 46(4), 913-921.

Dodge, R., \& Cline, T. S. (1901). The angle velocity of eye movements. Psychological Review, 8(2), 145.

Duchowski, A. T. (2007). Eye tracking methodology Theory and practice. London: Springer.

Dupont, L., Antrop, M., \& Van Eetvelde, V. (2014). Eyetracking Analysis in Landscape Perception Research: Influence of Photograph Properties and Landscape Characteristics. Landscape Research, 39(4), 417-432.

Dupont, L., Antrop, M., \& Van Eetvelde, V. (2015). Does landscape related expertise influence the visual perception of landscape photographs? Implications for participatory landscape planning and management. Landscape and Urban Planning, 141, 68-77. doi:http://dx.doi.org/10.1016/j.landurbplan.2015 .05 .003

EyeSee. (2015). Affordable Eye Tracking. Retrieved from http://eyesee-research.com/

Findlay, J. M., \& Gilchrist, I. D. (1998). Eye guidance and visual search. Eye guidance in reading and scene perception, 295-312.

GazeHawk. (2015). Webcam Eye Tracking. Retrieved from http://gazehawk.com/

Holmqvist, K., Nyström, M., Andersson, R., Dewhurst, R., Jarodzka, H., \& Van de Weijer, J. (2011). Eye tracking: A comprehensive guide to methods and measures. Oxford: Oxford University Press.

Holmqvist, K., Nyström, M., \& Mulvey, F. (2012). Eye tracker data quality: what it is and how to measure it. Paper presented at the Proceedings of the symposium on eye tracking research and applications. 
Incoul, A., Ooms, K., \& De Maeyer, P. (2015). Comparing Paper and Digital Topographic Maps Using Eye Tracking. In J. Brus, A. Vondrakova, \& V. Vozenilek (Eds.), Modern Trends in Cartography (pp. 339-356): Springer International Publishing.

Jacob, R., \& Karn, K. (2003). Eye tracking in humancomputer interaction and usability research: Ready to deliver the promises. In R. Radach, J. Hyona, \& H. Deubel (Eds.), The Mind's Eye: Cognitive and Applied Aspects of Eye Movement Research (pp. 573-605). Amsterdan: Elsevier.

Just, M. A., \& Carpenter, P. A. (1976). Eye fixations and cognitive processes. Cognitive Psychology, 8(4), 441-480.

Krassanakis, V., Filippakopoulou, V., \& Nakos, B. (2014). EyeMMV toolbox: An eye movement post-analysis tool based on a two-step spatial dispersion threshold for fixation identification. Journal of Eye Movement Research, 7(1), 1-10.

Mantiuk, R., Kowalik, M., Nowosielski, A., \& Bazyluk, B. (2012). Do-it-yourself eye tracker: Low-cost pupil-based eye tracker for computer graphics applications: Springer.

Nyström, M., Andersson, R., Holmqvist, K., \& van de Weijer, J. (2013). The influence of calibration method and eye physiology on eyetracking data quality. Behavior research methods, 45(1), 272288.

Ooms, K., Coltekin, A., De Maeyer, P., Dupont, L., Fabrikant, S. I., Incoul, A., . . V Van der Haegen, L. (2015). Combining user logging with eye tracking for interactive and dynamic applications. Behavior research methods, (in press). doi:10.3758/s13428-014-0542-3

Ooms, K., De Maeyer, P., Fack, V., Van Assche, E., \& Witlox, F. (2012). Interpreting maps through the eye of expert and novice users. International Journal of Geographical Information Science, 26(10), 1773-1788.

Pieters, R. (2008). A review of eye-tracking research in marketing. Review of marketing research, 4 , 123-147.

Poole, A., \& Ball, L. J. (2006). Eye tracking in human computer interaction and usability research: current status and future prospects. In C. Ghaoui (Ed.), Encyclopedia of Human Computer Interaction (pp. 211-219): Idea Group.

Popelka, S. (2014). Optimal eye fixation detection settings for cartographic purposes. 14th SGEM GeoConference on Informatics, Geoinformatics and Remote Sensing, 1(SGEM2014 Conference Proceedings, ISBN 978-619-7105-10-0/ISSN
Ooms, K., Dupont, L., Lapon, L., \& Popelka, S. (2015) Evaluation of the accuracy and precision of a low-cost eye tracking device pp.

Popelka, S., \& Brychtova, A. (2013). Eye-tracking Study on Different Perception of 2D and 3D Terrain Visualisation. The Cartographic Journal, 50(3), 240-246.

Pretorius, M. C., Calitz, A. P., \& van Greunen, D. (2005). The added value of eye tracking in the usability evaluation of a network management tool. Paper presented at the Proceedings of the 2005 annual research conference of the South African institute of computer scientists and information technologists on IT research in developing countries.

Rayner, K. (1998). Eye movement in reading and information processing: 20 years of research. Psychological Bulletin, 124(3), 372-422.

Rayner, K. (2009). Eye movements and attention in reading, scene perception, and visual search. The quarterly journal of experimental psychology, 62(8), 1457-1506.

Rayner, K., \& Castelhano, M. S. (2008). Eye movements during reading, scene perception, visual search, and while looking at print advertisements. Visual marketing: From attention to action, 942.

Reingold, E. M. (2014). Eye tracking research and technology: Towards objective measurement of data quality. Visual Cognition, 22(3-4), 635-652.

Sæther, L., Van Belle, W., Laeng, B., Brennen, T., \& Øvervoll, M. (2009). Anchoring gaze when categorizing faces' sex: evidence from eyetracking data. Vision Research, 49(23), 28702880.

Salvucci, D. D., \& Goldberg, J. H. (2000). Identifying fixations and saccades in eye-tracking protocols. Paper presented at the Proceedings of the 2000 symposium on Eye tracking research \& applications.

Schiessl, M., Duda, S., Thölke, A., \& Fischer, R. (2003). Eye Tracking and its Application in Usability and Media Research. MMI-interaktiv Journal - Online Zeitschrift zu Fragen der MenschMaschine-Interaktion, 6, 41-50.

SMI. (2012). Begaze Version 3.2 Manual. Retrieved from SMI. (2015). RED250 Technical Specification.

Retrieved from http://www.smivision.com/fileadmin/user uploa d/downloads/product flyer/prod_smi_red250 te chspecs.pdf

Strick, M., Holland, R. W., Van Baaren, R., \& Van Knippenberg, A. (2009). Humor in the eye tracker: Attention capture and distraction from 
context cues. The Journal of General Psychology: Experimental, Psychological, and Comparative Psychology, 137(1), 37-48.

TheEyeTribe. (2015a). The eye tribe tracker. Retrieved from https://theeyetribe.com/products/

TheEyeTribe. (2015b). Setting Up. Retrieved from http://dev.theeyetribe.com/start/

Tinker, M. A. (1946). The study of eye movements in reading. Psychological Bulletin, 43(2), 93.

Vansteenkiste, P., Cardon, G., D’Hondt, E., Philippaerts, R., \& Lenoir, M. (2013). The visual control of bicycle steering: The effects of speed and path width. Accident Analysis \& Prevention, 51, 222227.

Voßkühler, A., Nordmeier, V., Kuchinke, L., \& Jacobs, A. M. (2008). OGAMA (Open Gaze and Mouse Analyzer): open-source software designed to analyze eye and mouse movements in slideshow study designs. Behavior research methods, 40(4), 1150-1162.

Wass, S. V., Forssman, L., \& Leppänen, J. (2014). Robustness and Precision: How Data Quality May Influence Key Dependent Variables in Infant Eye-Tracker Analyses. Infancy, 19(5), 427-460.

Wolfe, J. M. (1994). Guided search 2.0 a revised model of visual search. Psychonomic Bulletin \& Review, 1(2), 202-238.

Wolfe, J. M. (2007). Guided search 4.0. Integrated models of cognitive systems, 99-119.

XLab. (2015). Xlabs, eye gaze and head via the webcam. Retrieved from http://xlabsgaze.com/

Yarbus, A. (1967). Eye Movements During Perception of Complex Objects Eye Movements and Vision (pp. 171-211): Springer US.

Zambarbieri, D., Carniglia, E., \& Robino, C. (2008). Eye Tracking Analysis in reading Online Newspapers. Journal of Eye Movement Research, 2(4), 1-8. 